\title{
COMPORTAMIENTO FISIOLÓGICO DE GRAMÍNEAS FORRAJERAS BAJO TRES NIVELES DE HUMEDAD EN CONDICIONES DE CASA MALLA
}

\section{PHISIOLOGY BEHAVIOR OF GRASSES UNDER THREE MOISTURE LEVELS IN SCREENHOUSE}

\author{
Liliana M. Atencio ${ }^{1}$, Jose J. Tapia ${ }^{1}$, Sergio L. Mejía ${ }^{2}$ y Jorge Cadena ${ }^{3}$
}

Recibido para publicación: Enero 30 de 2014 - Aceptado para publicación: Octubre 15 de 2014

\begin{abstract}
RESUMEN
La presente investigación tuvo como objetivo evaluar el efecto del nivel de humedad sobre el comportamiento fisiológico de diferentes gramíneas forrajeras. El experimento se realizó bajo condiciones de casa de malla en el Centro de Investigación Turipaná de Corpoica, (Cereté, Córdoba). Se utilizó un diseño de bloques completos al azar con 14 genotipos y tres (3) repeticiones. Se utilizaron tres regímenes de humedad, a manera de localidades dentro de la misma casa de malla. Dentro de las variables en estudio se encontraron: fotosíntesis neta, transpiración, conductancia estomática, potencial hídrico foliar, masa seca de hoja, área foliar, entre otras. Sólo las variables área foliar y potencial hídrico foliar presentaron interacciones significativas entre los factores Genotipo y Ambiente de humedad, mientras que el resto de variables, generalmente fueron afectadas por los factores principales $(\mathrm{p}<0.05)$. Los genotipos Bisset, Colosuana y Strikcland generaron la interacción para área foliar al presentar valores altos en condiciones de alta y media humedad. En el nivel de humedad bajo, las 14 gramíneas forrajeras presentaron los potenciales hídricos foliares más negativos. En este nivel se obtuvieron también los menores valores de conductancia estomática y transpiración y las menores acumulaciones de materia seca en todos los órganos, principalmente las hojas. BR02/1752 presentó la mayor acumulación de materia seca en las hojas (32,67 gplanta $\left.^{-1}\right)$, seguido de CIAT16051 (27,78 gplanta $\left.{ }^{-1}\right)$. Se concluye que el comportamiento fisiológico de las pasturas está determinado por las características genéticas y por el régimen de humedad implementado, y no por su interacción.
\end{abstract}

Palabras clave: Fisiología, potencial hídrico foliar, área foliar, B. pertusa cv. Colosuana, B. insculpta cv. Bisset

\begin{abstract}
The present study aimed to evaluate the effect of moisture level on the physiological behavior of different forage grasses. The experiment was conducted in screenhouse at the Center for Research Turipaná of Corpoica, (Cereté, Córdoba). Was used a design randomized complete block with 14 genotypes, and three (3) replications. Three moisture regimes were used in the manner of locations within the same screenhouse. Among the variables

\footnotetext{
1 Ingeniero Agrónomo, MsC. Investigador. Corporación Colombiana de Investigación Agropecuaria Cl. Turipaná, Montería, Colombia. 2 Ingeniero Agrónomo Ph.D. Investigador. Corporación Colombiana de Investigación Agropecuaria Cl. Turipaná, Montería, Colombia. Email: smejia@corpoica.org.co
} 
studied were: net photosynthesis, transpiration, stomatal conductance, leaf water potential, leaf dry mass, leaf area, among others. Only the variables leaf area and leaf water potential showed significant interactions between genotype and environment of moisture, while the other variables, were most affected by the main factors(p<0.05). Genotypes Bisset, Strikcland and Colosuana had an interaction for leaf area, showing the highest value at high and medium moisture. At low humidity, the 14 forage grasses had more negative leaf water potential. Low level moisture had the lowest values of stomatal conductance and transpiration and lower dry matter accumulation in all organs, mainly the leaves. BR02/1752 had the highest dry matter accumulation in leaves (32.67 gplant $\left.^{-1}\right)$ followed by CIAT16051 (27.78 gplant $\left.{ }^{-1}\right)$. It is concluded that the physiological behavior of pastures is determined by genetic characteristics and moisture regime, not by their interaction.

Key words: Phisiology, leaf area, leaf water potential, B. pertusa cv. Colosuana, B. insculpta cv. Bisset

\section{INTRODUCCIÓN}

El agua es uno de los factores de mayor importancia en el desarrollo de las gramíneas forrajeras, ya que esta constituye aproximadamente el $80 \%$ de los tejidos de las plantas. Cuando se presentan bajas precipitaciones o la distribución de estas es irregular, se generan condiciones de déficit de agua en el suelo, que afectan negativamente las relaciones hídricas internas, la fisiología y la morfología de las plantas forrajeras, lo cual limita la producción de forraje e incluso la supervivencia de las especies (Mattos et al. 2005a), disminuyendo las producciones de carne y leche en los sistemas ganaderos, ya que las gramíneas de pastoreo son la base de la alimentación.

Las gramíneas forrajeras al igual que muchas especies varían notablemente en su tolerancia a estrés por déficit hídrico. En algunos casos estas experimentan cambios que les puedan permitir adaptarse o escapar a los efectos negativos ocasionados por el estrés hídrico. Algunos de los aspectos en las plantas que se ven afectados por efectos del déficit hídrico son: la turgencia de los tejidos de las plantas, los procesos metabólicos y reacciones bioquímicas, la absorción de los nutrientes, la translocación de sustancias orgánicas e inorgánicas, entre otros.

A nivel de casa malla se han realizado diversos estudios que permiten observar los efectos que ocasiona el déficit hídrico en las gramíneas forrajeras, muchos realizados principalmente con especies del género Brachiaria. Entre estas cabe mencionar las desarrolladas por Santos et al. (2013), quienes observaron que los genotipos Brachiaria brizantha cv. Piatá y Brachiaria brizantha cv. Marandú disminuyeron la producción de la materia seca aérea y el área foliar e incrementaron el porcentaje de raíces en las plantas bajo condiciones de déficit hídrico.

Mattos (2005a) observó que Brachiaria brizantha tolera condiciones de déficit hídrico, debido a una menor sensibilidad de sus estomas al estrés, menor comprometimiento de sus tasas fotosintéticas y transpiración, altas tasas de elongación foliar, bajas tasas de senescencia de sus laminas foliares y alta producción de raíces Por su parte, Guenni y colaboradores (2002a, 2002b), presentaron que los valores de potencial hídrico foliar disminuyeron a valores entre $-1,5$ y $-2 \mathrm{MPa}$ en Brachiaria mutica y entre -2,5 a -3 MPa en Brachiaria decumbens, Brachiaria humidicola y Brachiaria dyctioneura, 
fueron las especies más tolerantes a la sequía debido a la profundización de sus raíces.

Teniendo en cuenta que las investigaciones desarrolladas se limitan a un número determinado de géneros, se desarrolló el presente estudio que tuvo como objetivo evaluar el efecto del déficit de humedad en el comportamiento fisiológico de diferentes gramíneas forrajeras bajo condiciones de casa de malla, que sirva de base para la selección de nuevos materiales, que tengan mayor eficiencia en el uso del agua, sobre todo en la época seca, que le permita al ganadero disponer de forraje durante este periodo.

\section{MATERIALES Y MÉTODOS}

\section{Localización}

El experimento se realizó bajo condiciones de casa de malla en el Centro de Investigación Turipaná de Corpoica, ubicado en el municipio de Cereté, departamento de Córdoba. Durante el periodo experimental se presentó en promedio una temperatura de $31^{\circ} \mathrm{C}$ y humedad relativa de $61,5 \%$.

\section{Diseño experimental}

El experimentó se desarrolló bajo un diseño de bloques completos al azar con 14 genotipos y tres repeticiones. El experimento se realizó bajo tres (3) ambientes de humedad (alto, medio, bajo), a manera de localidades dentro de la misma casa de malla, estableciendo un conjunto de tres experimentos completos con el mismo número de tratamientos (genotipos). Esta distribución se utilizó para evitar que las Iluvias frecuentes, acompañadas de vientos fuertes, puedan afectar los experimentos.
Los genotipos correspondieron a gramíneas forrajeras de los géneros: Brachiaria ( $B$. brizantha CIAT26124, B. brizantha CIAT26990, B. brizantha cv. Piatá, B. brizantha cv. Toledo, $B$. humidicola CIAT 16888, $B$. hibrido BR02/1752, B. hibrido BR02/1794 y B. hibrido Cv. Mulato II), Botrhiochloa (B. pertusa cv. Colosuana y $B$. insculpta cv. Bisset), Panicum (P. maximum CIAT16051, $P$. maximum CIAT6799 y $P$. maximum cv. Mombaza) y Digitaria (D. milanjiana Cv. Strickland). La mayoría de los genotipos fueron introducidos de África a través del Centro Internacional de Agricultura Tropical (CIAT).

Para el desarrollo del ensayo se tomó suelo de la finca "Altamonte", ubicada en el municipio de Sahagún, Córdoba, la cual fue seleccionada por presentar un sistema de producción ganadero típico de la zona y ser representativa de la subregión Sabanas del Caribe, en específico de las Sabanas Colinadas de Córdoba, Sucre y Bolívar. Se realizó análisis de suelo en el Laboratorio de Suelos y Aguas de la Universidad de Córdoba, el cual indicó las siguientes características: textura francoarenosa, $\mathrm{pH}$ de 5,9; contenidos medios de calcio. (4 $\mathrm{cmol}^{+} \mathrm{kg}^{-1}$ de suelo), magnesio alto $\left(4,5 \mathrm{cmol}^{+} \mathrm{kg}^{-1}\right.$ de suelo), potasio bajo $(0,06$ $\mathrm{cmol}^{+} \mathrm{kg}^{-1}$ de suelo); fósforo $\left(8,3 \mathrm{mg} \mathrm{kg}^{-1}\right)$ alto contenido de Fe $\left(118,8 \mathrm{mg} \mathrm{kg}^{-1}\right)$.

Se seleccionaron macollas uniformes de eras de multiplicación de los diferentes materiales genéticos, las cuales se sembraron en bandejas plásticas de germinación por un periodo de tres semanas, con el fin de promover el desarrollo de raíces y uniformizar el material vegetativo a trasplantar a las macetas. Posteriormente, se seleccionaron macollas uniformes en 
tamaño y se realizó el trasplante a las macetas plásticas, que fueron previamente llenas con $3,0 \mathrm{~kg}$ de suelo uniformizado. Al momento del trasplante se realizó la fertilización con base en $150 \mathrm{~kg}$ de nitrógeno, $46 \mathrm{~kg}$ de fósforo, $30 \mathrm{~kg}$ de potasio y elementos menores, correspondientes a $20 \mathrm{~kg}$ de vicor por hectarea; los fertilizantes fueron disueltos en agua $y$ aplicados proporcionalmente al suelo de cada maceta, antes de la siembra. Posteriormente, las plántulas se dejaron crecer por un período de tres semanas, suministrando humedad suficiente (capacidad de campo) para promover el establecimiento de las mismas. Transcurrido este periodo se inició la aplicación de los tres ambientes de humedad.

Los ambientes de humedad implementados fueron: 1) Alta humedad, el suelo se mantuvo a capacidad de campo, durante todo el periodo experimental. 2) Humedad Media, después del periodo de establecimiento el suelo se mantuvo con el $50 \%$ de la capacidad de campo hasta el final del experimento. 3) Baja humedad, la aplicación de agua a las macetas se suspendió después del periodo de establecimiento, hasta el final del experimento, que correspondió a 40 días.

Para aplicación de los ambientes de humedad, se determinó el contenido de humedad a capacidad de campo y punto de marchitez permanente. Para la determinación del contenido de humedad a capacidad de campo, se utilizó el método gravimétrico según la metodología descrita por Earl (2003), en la que se registró una humedad del 32\%, considerada como el contenido de humedad a capacidad de campo. Para la obtención del punto de marchitez permanente se empleó el método biológico o de invernadero, descrito por Montenegro y Malagón (1990) y Gurovich (2000), por medio del cual se registró una humedad del 13\%, considerado punto de marchitez permanente. Con base en lo anterior, se determinó el agua aprovechable (CC-PMP) y el ambiente de humedad correspondiente al $50 \%$ del agua aprovechable (22\% de contenido de humedad).

Con el fin de mantener el contenido de humedad indicado para cada uno de estos ambientes, diariamente se determinó el contenido de humedad del suelo con la ayuda del determinador $\mathrm{HH} 2$ Delta-T Devices (Cambridge, UK). El equipo, permitió el cálculo automático de la cantidad de agua a aplicar en cada matera, con base en la textura (francoarenosa), volumen y densidad aparente del suelo, frente al porcentaje de humedad actual.

A los 40 días después del establecimiento, se realizaron mediciones de intercambio gaseoso conelusodeunmedidorportátilmodeloCIRAS-2 de la PP Systems International, Inc. (Amesbury, MA, USA). Las mediciones se realizaron en una hoja fotosintéticamente activa, sin daños mecánicos, ubicada en el estrato medio y conservando su posición original. Las lecturas se hicieron entre las 8:00 y las 10:00 a.m., con una radiación fotosintéticamente activa de $1200 \mu$ moles fotonesm ${ }^{-2} \mathrm{~s}^{-1}$ y concentraciones de $\mathrm{CO}_{2}$ en el rango de 350-380 ppm y de temperatura de $27,1-35,4{ }^{\circ} \mathrm{C}$. Las variables determinadas fueron: transpiración (mmoles $\mathrm{H}_{2} \mathrm{O} \mathrm{m}^{-2} \mathrm{~s}^{-1}$ ), conductancia estomática ( $\mu$ moles $\mathrm{CO}_{2} \mathrm{~m}^{-2} \mathrm{~s}^{-1}$ ) y fotosíntesis neta ( $\mu$ moles $\mathrm{CO}_{2}$ $\mathrm{m}^{-2} \mathrm{~s}^{-1}$ ). Así mismo, se determinó el potencial hídrico foliar (bares) empleando la cámara de presión (Scholander et al. 1965), con el uso 
de una bomba de presión modelo 3005 HGPL (Soil Moisture Equipment Corp., Santa Barbara, CA, USA).

Por último se realizó un muestreo destructivo, en el que se extrajeron las plantas completas y se separaron en hojas, tallos y raíces, para determinar las variables masa seca, relación hoja/tallo, longitud total de raíz (m), área foliar $\left(\mathrm{cm}^{2}\right)$ y área foliar específico $\left(\mathrm{cm}^{2} \mathrm{~g}^{-1}\right)$.

La longitud total de la raíz, se determinó extrayendo la totalidad de las raíces, y una vez lavadas, fueron escaneadas y analizadas con el programa WinRhizo Reg (Regent Instruments Canada INC.). Para la masa seca, las muestras fueron sometidas a secado en una estufa a $60{ }^{\circ} \mathrm{C}$ por 72 horas y posteriormente pesadas. A partir de los pesos de hoja y tallo, se determinó la relación de hoja:tallo. El área foliar específica, se calculó dividiendo el área foliar sobre el peso seco de las hojas.

\section{Análisis estadístico}

El procesamiento estadístico de los datos se realizó mediante el paquete estadístico SAS V9,0 (Statistical Analysis System), realizando un análisis combinado de los experimentos, asumiendo los ambientes de humedad como localidades del mismo experimento (como usualmente se hace en evaluaciones de adaptación de genotipos, en diferentes ambientes). En los casos en los cuales se detectaron diferencias significativas en los análisis de varianza $(\mathrm{p}<0.05)$, se realizó una prueba de comparación de medias mediante la prueba de HSD de Tukey.

\section{RESULTADOS Y DISCUSIÓN}

La mayor parte de las variables evaluadas en estos experimentos fueron afectadas por los factores principales Genotipo y Ambiente de humedad ( $\mathrm{p}<0.05)$ (Tabla 1 y 2). Sólo las variables área foliar y potencial hídrico foliar, presentaron interacciones significativas entre los genotipos y los ambientes de humedad. Esto indica que las mayores diferencias en este experimento, se presentaron por efecto a las características genéticas de las pasturas y por el régimen de humedad implementado.

Materia seca. En la tabla 1 se presenta la materia seca acumulada en cada uno de los órganos de la planta, para cada genotipo. Se observa que el genotipo BR02/1752 se resalta por presentar la mayor cantidad de materia seca acumulada en las hojas, con 32,67 g planta $^{-1}$ seguido del genotipo CIAT16051 con 27,78 g planta $^{-1}$. En cuanto a la materia seca acumulada en los tallos, los mayores valores se presentan en los genotipos Colosuana, BR02/1752 y Piatá con valores de 48,67; 46,00 y 42,44 g planta $^{-1}$, respectivamente. En raíces se destacan los genotipos Strickland con 60,85 g planta $^{-1}$ y CIAT16051 con 46,51 g planta $^{-1}$. Con los anteriores resultados, los materiales genéticos con la mayor cantidad de materia seca acumulada en la totalidad de la planta fueron Strickland con 116,84 g planta $^{-1}$, BR02/1752 con 108,88 g planta ${ }^{-1}$ y CIAT16051 con 104,03 g planta $^{-1}$.

Con relación al efecto de los ambientes de humedad sobre la acumulación de materia seca en las pasturas, se observó un mayor crecimiento y acumulación de materia seca todos los órganos de la planta generalmente 
en el nivel de humedad alto y medio (Tabla 2). De acuerdo con estos resultados, la mayor disponibilidad de humedad en estos niveles, favoreció la división y elongación celular, al igual que el metabolismo, propiciando un mayor desarrollo y crecimiento en la planta, favorecido por la hidratación y la turgencia en las células generada por la oferta de agua en el suelo (Vila 2011; Jarma et al. 2012). Los resultados obtenidos coinciden con los encontrados por García (2012) y Bahrami et al. (2010), quienes observaron que la producción de masa seca incrementa en ambientes de humedad con mayor disponibilidad de agua (cercana a la presente en capacidad de campo).

Relación Hoja:Tallo. En pastos y forrajes, el interés del ganadero se centra en la acumulación de la materia seca que ocurre en las hojas, pues es la parte de la planta que es consumida en mayor proporción por el animal y la que aporta el mayor contenido de proteínas y nutrientes. Así mismo, tiene en cuenta la relación hoja:tallo a fin de determinar la proporción de asimilados destinados a las hojas. Bajo estas condiciones, desde el punto de vista de los aportes al sistema ganadero, es deseable una alta relación hoja:tallo, es decir, que la mayor proporción de asimilados se destinen a la producción de hojas, lo cual puede ser asociado con la calidad de la pastura.

Los resultados obtenidos en estos experimentos, muestran que las mayores relaciones hoja:tallo, se presentaron en los genotipos CIAT16051, Mombaza, Mulato y Toledo. Estos presentaron valores de 0,98; 0,88; 0,86 y 0,81 respectivamente. Lo anterior indica que por cada parte de materia seca acumulada en el tallo, aproximadamente se acumularon
$0,98,0,88,0,86$ y 0,81 partes en las hojas de los genotipos CIAT16051, Mombaza, Mulato y Toledo, respectivamente (Tabla 1), lo cual puede favorecer la calidad nutricional de estas gramíneas. Según Araya y Boschini (2005), las pasturas con mayor proporción de hojas presentan mayor contenido de proteína, confiriéndole mejor calidad nutricional a la pastura.

Con relación a los ambientes de humedad, las mayores relaciones hoja:tallo se obtuvieron en el nivel de humedad alto con 0,87 ; seguido del nivel de humedad bajo con 0,73 y nivel medio con 0,59 (Tabla 2). Según estos resultados, las pasturas en estudio, bajo condiciones de humedad media, presentan una mejor condición para la alimentación ganadera, debido a que se favorece la distribución de asimilados hacia las hojas. Las buenas condiciones de aireación del suelo en los niveles medios de humedad, adicional a la adecuada disponibilidad de humedad, probablemente favorecen el crecimiento de la parte aérea de las pasturas y un menor porcentaje de desprendimiento y senescencia de las hojas (Mattos et al. 2005b).

Longitud total de raíz. Esta es una característica de alta importancia, dado que se relaciona con la tolerancia a déficit hídrico. Los resultados de estos experimentos indicaron una mayor longitud de raíces en el genotipo BR02/1794 con una longitud total de raíces de 125,74 m y los menores promedios se presentaron en los genotipos Bisset con 7,38 m y CIAT26990 con 9,99 m (Tabla 1). Lo anterior indicaría, probablemente una mayor capacidad de tolerancia al déficit hídrico en el genotipo BR02/1794, ya que existiría una mayor 


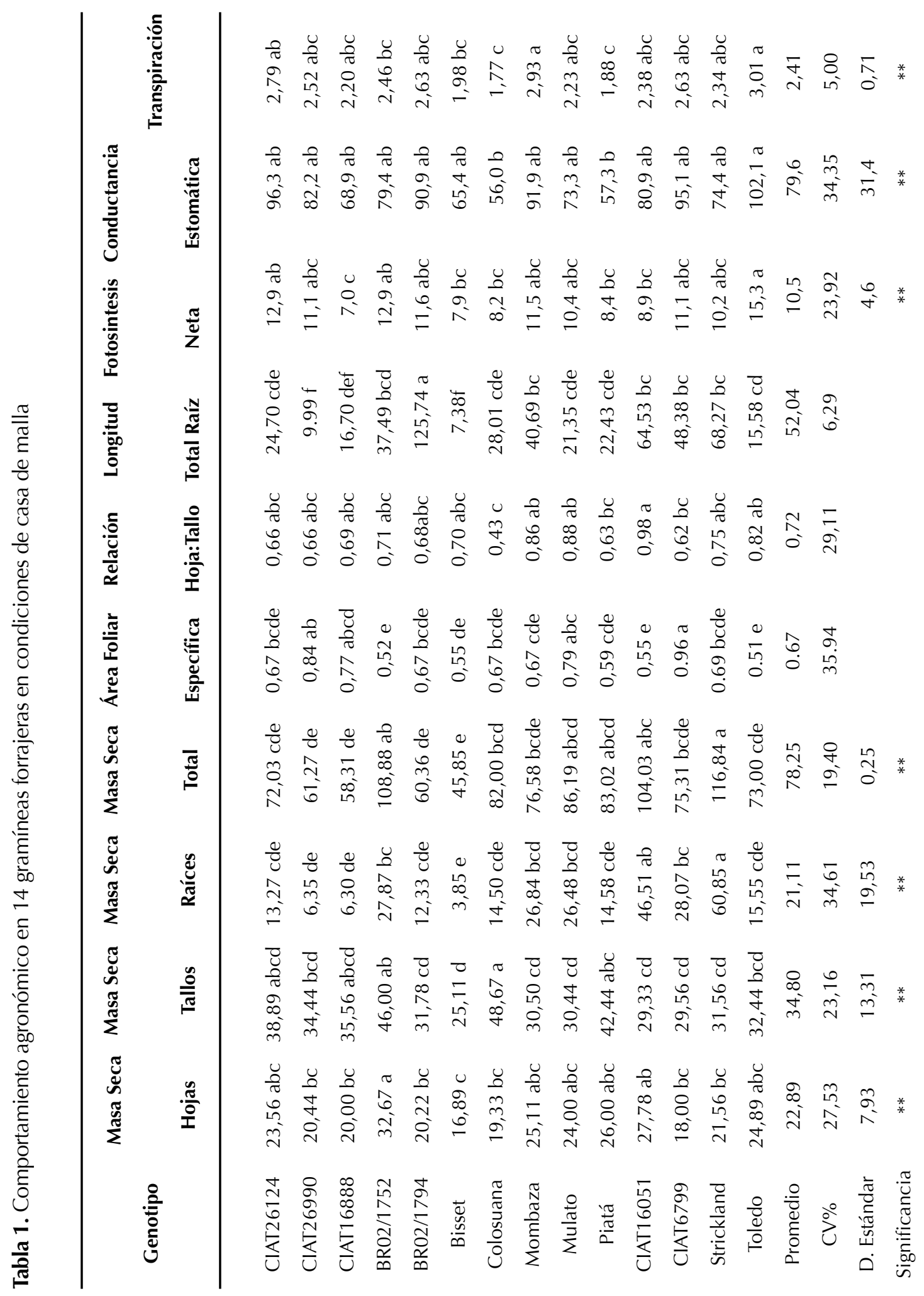




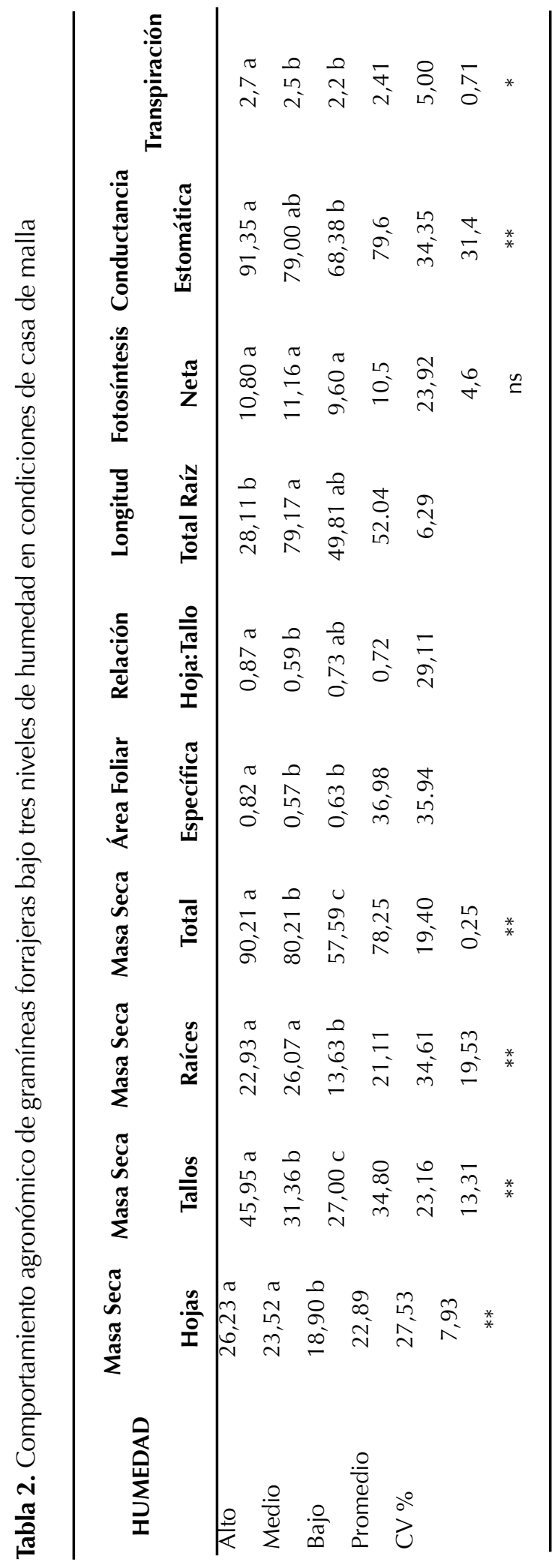

exploración de volumen de suelo, facilitando la extracción de agua existente en las capas inferiores (Khan et al. 2010).

Con relación a los niveles de humedad, se observó que en el nivel de humedad medio y bajo, las pasturas presentaron la mayor longitud total de raíces con 79,17 y 49,81 m (Tabla 2). En la literatura está bien documentado el hecho de que las plantas presentan un mayor crecimiento de las raíces bajo condiciones de estrés por humedad. Esto se debe a la necesidad de la planta por explorar un mayor volumen de suelo y establecer una mayor superficie de contacto entre la raíz y el suelo (Blum 2011). Es de aclarar que esta mayor exploración del suelo se presenta en las plantas hasta cuando las condiciones de estrés sean tan severas, que causen inhibición en el crecimiento y suspensión del desarrollo del sistema radicular de las pasturas (Ontiveros et al. 2005, Hartung 1996).

Área foliar específica. Esta variable es una aproximación cuantitativa para estimar la dureza de la hoja y por tanto su palatabilidad. Entre más área foliar desarrolle una pastura por unidad de peso, más tierna y más digerible es para el animal. Desde el punto de vista productivo, conviene tener gramíneas que presenten una alta área foliar especifica.

En la tabla 1 se observa que el genotipo CIAT6799 presenta el valor más alto de área foliar especifica con $0,96 \mathrm{~cm}^{2} \mathrm{~g}^{-1}$, seguido de CIAT26990 con 0,84 $\mathrm{cm}^{2} \mathrm{~g}^{-1}$, Mulato II con $0,79 \mathrm{~cm}^{2} \mathrm{~g}^{-1}$ y CIAT16888 con $0,77 \mathrm{~cm}^{2} \mathrm{~g}^{-1}$.

Por el contrario pasturas como Toledo presentan valores más bajos con $0,51 \mathrm{~cm}^{2} \mathrm{~g}^{-1}$, BR02/1752 con 0,52 $\mathrm{cm}^{2} \mathrm{~g}^{-1}$ y CIAT16051 con $0,55 \mathrm{~cm}^{2} \mathrm{~g}^{-1}$ (Tabla 2). 
Estos resultados indican que desde el punto de vista de la palatabilidad, los materiales CIAT6799, CIAT26990, Mulato y CIAT16888 serían los más convenientes para la alimentación animal. Algunos autores (Garnier y Laurent 1994; Pérez et al. 2004) sostienen que la composición química del forraje (producción de almidón, compuestos secundarios) y la anatomía de la planta (tamaño de las células, grosor de las paredes celular, presencia de tejidos vasculares, mesófilo), entre otros factores, determinan un mayor espesor y una mayor palatabilidad de la pastura.

Con relación a los niveles de humedad, se observó que el área foliar específica fue mayor en el nivel de humedad alto con un promedio de $0,82 \mathrm{~cm}^{2} \mathrm{~g}^{-1}$, seguido por el nivel bajo $\mathrm{y}$ medio con 0,63 y $0,57 \mathrm{~cm}^{2} \mathrm{~g}^{-1}$, respectivamente (Tabla 2).

Los mayores valores en el nivel de humedad alto, indican que a este nivel de humedad las hojas de las gramíneas forrajeras son más tiernas y palatables. Bajo condiciones de niveles de humedad medio y bajo, la reducción en la turgencia de las células que genera un reforzamiento estructural, acumulación de compuestos basados en macromoléculas como taninos, sílice, ligninas o fibras de celulosa y hemicelulosa y al decrecimiento de los porcentajes de carbohidratos simples y grasas (Baeza 2002), dan lugar a estructuras más compactas y gruesas (paredes celulares, parénquimas, cutículas), así como células y espacios intercelulares de menor tamaño (Herralde 2000).

Los resultados obtenidos en esta investigación coinciden con Balaguera et al. (2008), quienes encontraron una relación directamente proporcional entre el agua suministrada y el área foliar específica. Cuando las plantas se someten a déficit hídrico, presentan cambios en el contenido de sus carbohidratos solubles y en la actividad de las enzimas implicadas en el metabolismo del carbono.

Lo anterior se asocia con la calidad de las pasturas, expresada como contenidos de fibras, en especial fibra de detergente ácido (FDN, que involucra celulosa, hemicelulosa y lignina) y puede ser un indicativo de la baja calidad durante los periodos de sequía.

Área foliar. El crecimiento foliar es uno de los primeros procesos fisiológicos que es afectado por la sequía, debido a los efectos del agua sobre la expansión celular. Los resultados obtenidos en este experimento indicaron interacciones significativas entre genotipos y niveles de humedad. Al observar el comportamiento de las 14 gramíneas forrajeras bajo cada uno de los ambientes de humedad (Figura 1). Se observó que los genotipos Bisset, Colosuana y Strikcland presentaron diferencias estadísticas entre los niveles de humedad, generando así una interacción.

Los mayores valores de área foliar para estas tres gramíneas forrajeras se presentaron en condiciones de alta y media humedad.

En el nivel de humedad alto, se obtuvieron valores de 16,25 $\mathrm{cm}^{2}$ para Bisset, 18,06 $\mathrm{cm}^{2}$ para Colosuana y $36,11 \mathrm{~cm}^{2}$ para Strikcland. En el nivel de humedad medio los valores obtenidos fueron $8,06 \mathrm{~cm}^{2}$ para Bisset, $14,45 \mathrm{~cm}^{2}$ para Colosuana y $10,33 \mathrm{~cm}^{2}$ para Strikcland. 


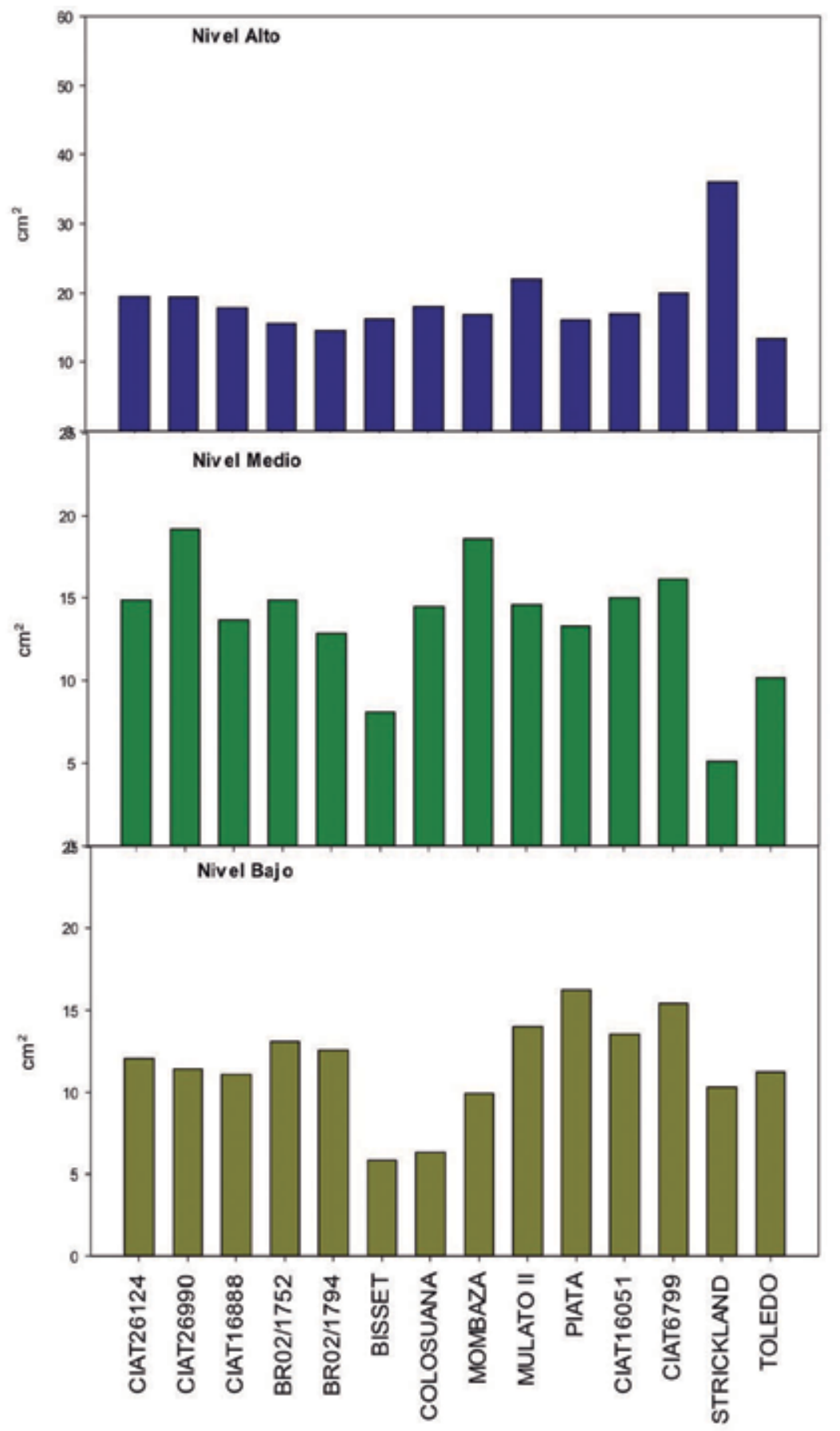

Lo anterior indica que la disponibilidad de humedad proporcionada por estos ambientes favorece la turgencia requerida para el crecimiento de estas gramíneas forrajeras. El agua a nivel celular se requiere para mantener una mayor turgencia, la cual está asociada al crecimiento y elongación celular, a la vez que menor senescencia de las láminas foliares (Mattos et al. 2005a; Azam y Squire 2002).

Desde el punto de vista productivo, es conveniente la selección de pasturas, que bajo 
condiciones de estrés por humedad, puedan mantener el crecimiento foliar y la producción de forraje (ser más estables).

En este sentido se destaca el genotipo CIAT6799, por presentar valores superiores a la media general de área foliar $\left(14,67 \mathrm{~cm}^{2}\right)$.

Potencial hídrico. Se presentó una interacción significativa entre los niveles de humedad y los genotipos $(p<0.05)$, indicando un efecto diferencial de los genotipos en los niveles de humedad.

En la Figura 2, se puede observar que en el nivel de humedad bajo, las 14 gramíneas forrajeras presentaron estadísticamente potenciales hídricos foliares más negativos, indicando el grado de deshidratación de la planta como producto de la resistencia de flujo de agua del suelo hacia las raíces (Rodríguez et al. 2009).

Dada la variabilidad que se presenta en el comportamiento de los 14 genotipos bajo los tres ambientes de humedad, se pueden resaltar los genotipos con la mejor condición de hidratación bajo cada ambiente. Para el nivel de humedad bajo, convienen genotipos que a pesar de la baja disponibilidad de agua, puedan mantener potenciales hídricos más altos (menos negativos), que aseguren la actividad metabólica de la planta.

En la investigación, se destacan los genotipos CIAT6799 con valores de -1,24 MPa, Toledo con -1,24 MPa y CIAT26124 con -1,25 MPa, por presentar estadísticamente el potencial hídrico foliar menos negativo en el nivel bajo, indicando que estos genotipos pudieron mantener un mayor grado de turgencia de las células bajo condiciones limitantes de humedad.
Lo anterior, indica que estos genotipos pueden mantener la actividad de los procesos fisiológicos y el crecimiento bajo condiciones limitadas de humedad.

En el nivel de humedad medio, los valores menos negativos de potencial hídrico foliar se observaron en los genotipos CIAT16888 con -0,9 MPa, CIAT26124 con -0,98 MPa y CIAT6799 con -0,98 MPa y en el nivel de humedad alto, se resalta el genotipo BR02/1794 con -0,83 MPa por presentar el mayor potencial hídrico foliar.

Por el contrario, el género Botrhiochloa con los genotipos Bisset y Colosuana, presentaron los potenciales hídricos más negativos, principalmente en el nivel de humedad bajo, lo que conllevó a que fueran genotipos menos tolerantes a las condiciones de sequía.

Intercambio gaseoso. Los parámetros de intercambio gaseoso son procesos fisiológicos que pueden ser afectados por el déficit hídrico, debido al efecto del agua sobre los movimientos estomáticos y funcionamiento del aparato fotosintético en general. En este caso, no se presentaron interacciones significativas entre genotipos y los niveles de humedad. Se destacan por valores superiores de fotosíntesis neta los genotipos CIAT26124, CIAT26990, BR02/1752, BR02/1794, Mombaza, Mulato II, CIAT6799, Strickland y Toledo. Estos genotipos en general, presentaron también los mayores valores de conductancia estomática y transpiración, indicando una actividad plena de los procesos fotosintéticos. En especial se destaca Toledo por presentar numéricamente los valores más altos de fotosíntesis neta con 15,3 $\mu \mathrm{mol}$ de $\mathrm{CO}_{2} \mathrm{~m}^{-2} \mathrm{~s}^{-1}$. Lo anterior se debe a sus 


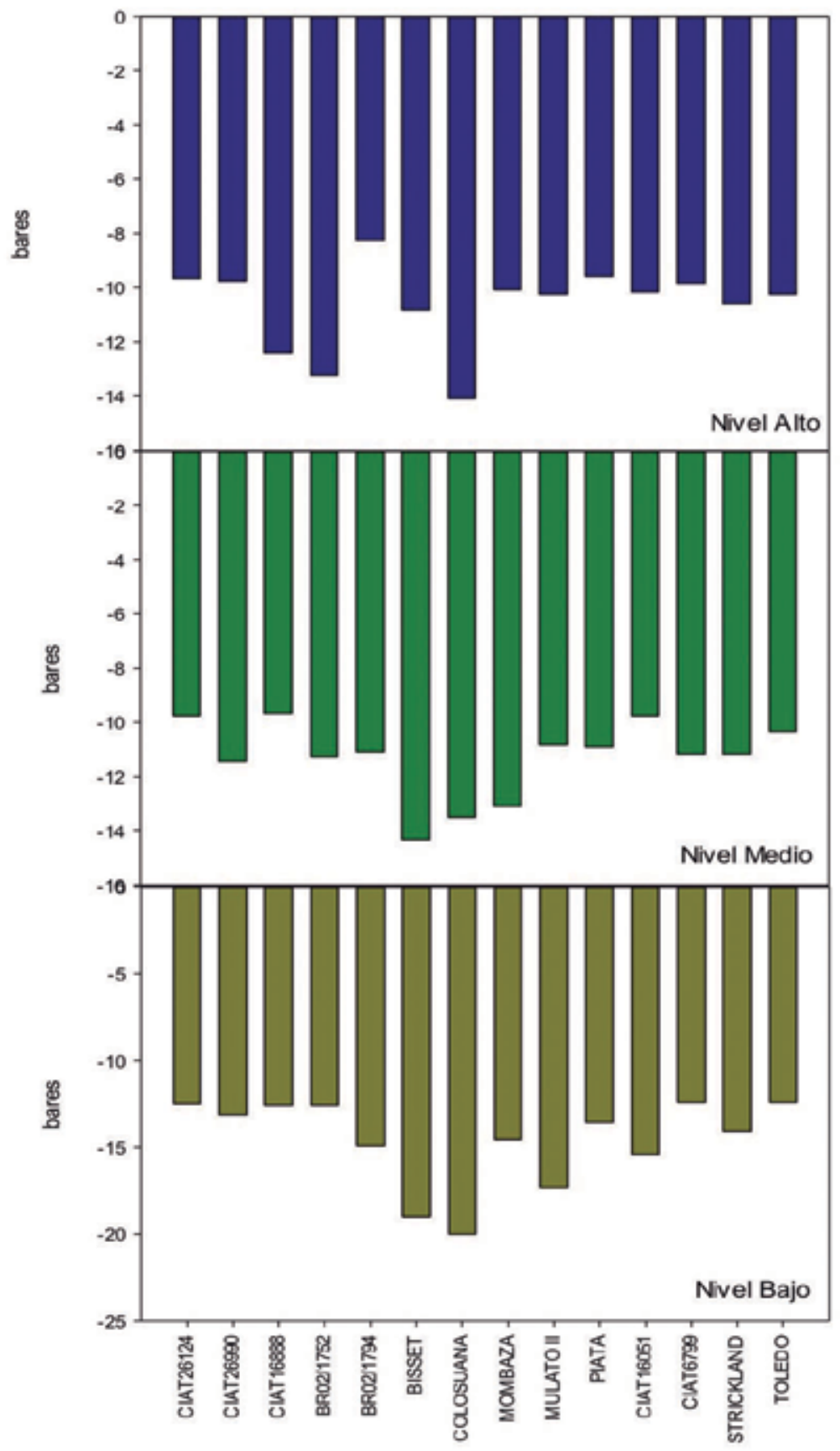

valores superiores de conductancia estomática de 102,1 $\mu$ moles $\mathrm{CO}_{2} \mathrm{~m}^{-2} \mathrm{~s}^{-1}$ y transpiración de 3,01 mmoles $\mathrm{H}_{2} \mathrm{Om}^{-2} \mathrm{~s}^{-1}$, indicando una mejor condición de hidratación (Tabla 1).

Dentro de los promisorios, se resaltan los genotipos CIAT26124 con una fotosíntesis neta de $12,9 \mu \mathrm{mol}$ de $\mathrm{CO}_{2} \mathrm{~m}^{-2} \mathrm{~s}^{-1}$, conductancia estomática de 96,3 umoles $\mathrm{CO}_{2} \quad \mathrm{~m}^{-2} \mathrm{~s}^{-1} \mathrm{y}$ transpiración de 2,79 mmoles $\mathrm{H}_{2} \mathrm{O} \mathrm{m} \mathrm{m}^{-2} \mathrm{~s}^{-1}$. Así mismo BR02/1752 al presentar una fotosíntesis neta de $12,9 \mu \mathrm{mol}$ de $\mathrm{CO}_{2} \mathrm{~m}^{-2} \mathrm{~s}^{-1}$.

Por el contrario, los menores promedios en conductancia estomática y transpiración se presentaron en Colosuana con 56,0 $\mu$ moles 
$\mathrm{CO}_{2} \mathrm{~m}^{-2} \mathrm{~s}^{-1}$ y 1,77 mmoles $\mathrm{H}_{2} \mathrm{Om}^{-2} \mathrm{~s}^{-1}$, respectivamente, seguido de Piatá con una conductancia estomática de 57,3 $\mu$ moles $\mathrm{CO}_{2} \mathrm{~m}^{-2} \mathrm{~s}^{-1}$ y transpiración de 1,88 mmoles $\mathrm{H}_{2} \mathrm{Om}^{-2} \mathrm{~s}^{-1}$. Mientras que la menor fotosíntesis neta se presentó en el genotipo CIAT16888 con $7,0 \mu \mathrm{mol}$ de $\mathrm{CO}_{2} \mathrm{~m}^{-2} \mathrm{~s}^{-1}$.

Con relación a los niveles de humedad se observaron efectos sobre la conductancia estomático y la transpiración, mas no sobre la fotosíntesis (Tabla 2).

En el nivel de humedad bajo, se presentaron los menores valores de conductancia estomática y transpiración $\left(2,7\right.$ mmoles $\mathrm{H}_{2} \mathrm{Om}^{-2} \mathrm{~s}^{-1}$ y 91,35 umoles $\mathrm{CO}_{2} \mathrm{~m}^{-2} \mathrm{~s}^{-1}$, respectivamente), debido a la baja disponibilidad de agua. La fotosíntesis no fue afectada debido a que bajo condiciones de escases de humedad en el suelo presentadas durante el experimento, posiblemente se produjo un cierre parcial de estomas, lo que afectó más la difusión del vapor de agua que de $\mathrm{CO}_{2}$. El déficit hídrico produce pérdida de turgencia de las células guardas de los estomas, induciendo un cierre parcial de éstos y por ende una disminución en la transpiración. El cierre parcial de los estomas afecta más la difusión del vapor de agua que la difusión del $\mathrm{CO}_{2}$.

Otras investigaciones han mostrado resultados similares, por ejemplo Hoyos (2007), en un experimento muy similar, encontró que la sequía sólo afectó las tasas de transpiración y conductancia estomática en gramíneas forrajeras, pero no la fotosíntesis. A este respecto, algunos autores precisan que la disminución del contenido de humedad del suelo, influye en el grado de apertura estomática, afectando más el proceso de transpiración que el de fotosíntesis (Barroso y Jérez 2000; Passioura 2002).

\section{CONCLUSIONES}

1. La mayor acumulación de materia seca se observó en el nivel de humedad alto, para todos los órganos de la planta.

2. En cuanto a las variables de mayor importancia para el ganadero, el genotipo BR02/1752, presentó la mayor acumulación de materia seca de hojas, pero simultáneamente altas producciones de materia seca de tallos, lo cual redujo su relación hoja:tallo.

3. Los genotipos CIAT16051, Mombaza, Mulato y Toledo presentaron las mayores relaciones de hoja:tallo.

4. El genotipo BR02/1794 fue el genotipo con la mayor longitud de las raíces.

5. Las gramíneas forrajeros CIAT6799, CIAT26990, Mulato II y CIAT16888 presentaron los valores más altos de área foliar específica.

6. Los genotipos CIAT26124, CIAT26990, BR02/1752, BR02/1794, Mombaza, Mulato II, CIAT6799, Strickland y Toledo se caracterizan por presentar una actividad plena de los procesos fotosintéticos.

7. La conductancia estomática yla transpiración presentaron mayor sensibilidad al déficit hídrico y los mayores valores en el nivel de humedad alto.

8. El potencial hídrico puede ser una variable fisiológica, que ayude en la selección de genotipos, más eficientes en el uso del agua. 


\section{REFERENCIAS}

Azam, S. and Squire, G. 2007. Principles of Tropical Agronomy. CABI Publishing.

Araya, M. y Boschini, C. 2005. Producción de forraje y calidad nutricional de variedades de Pennisetum purpureum en la meseta central de Costa Rica. Agronomía Mesoamericana. 16(1): 37-43.

Baeza, S. 2002. Área específica foliar como variable de respuesta al pastoreo en gramíneas dominantes de una pradera natural. Facultad de Ciencias, Universidad de la República. 17p.

Bahrami, H., Jafar, M., and Kamgar, A. 2010. Effect of water stress on ten forage grasses native or introduced to Iran. Japanese Society of Grassland Science. pp: 2

\section{Balaguera, H., Álvarez, J. y Rodríguez, J.} 2008. Efecto del déficit de agua en el trasplante de plántulas de tomate (Solanum lycopersicum L.). Agronomía Colombiana 26(2): 246-255.

Barroso, L. 2000. Comportamiento de las relaciones hídricas en la albahaca blanca (Ocimum basilicum L.) al ser irrigadas con diferentes volúmenes de agua. CultivosTropicales 21(3):57-59

Blum, A. 2011. Plant Stress and Plant Production. In: Plant Breeding for Water-Limited Environments, Hardcover. p11-52.

Earl, H. 2003. A precise gravimetric method for simulating drought stress in potexperiments. Crop Science 43(5):1868-1873.
García, S. 2012. Efecto del estrés hídrico sobre el crecimiento y habilidad competitiva de Lolium perenne L. y Bromus valdivianus Phill. Tesis de pregrado. Universidad Austral de Chile. Valdivia - Chile. 49p

Garnier B. and Laurent G. 1994. Leaf anatomy, specific mass and water content in congeneric annual and perennial grass species. New Phytol, 128. p725-736.

Guenni, O., Marín, D. and Baruch, Z. 2002. Responses to drought of five Brachiaria species. I. Biomass production, leaf growth, root distribution, water use and forage quality. Facultad de Agronomía. Plant and soil 243: 229-241.

Guenni, O., Baruch, Z. and Marín, D. 2002. Responses to drought of five Brachiaria species. II. Water relations and leaf gas exchange. Facultad de Agronomía. Plant and soil 243: 229-241.

Gurovich, L. 2000. Riego Superficial Tecnificado. Tercera Edición ampliada. Colección Textos Universitarios Pontificia Universidad Católica de Chile. 635 p. ISBN 956-14-0476-1

Hartung, W. 1996. Trockenheit. En: Brunold,CH.; Rüegesegger, A.; Brändle, R. edit.Stress bei Pflanzen. UTB für Wissenschaft, Verlag Paul Haupt. Stuttgart. p199-132.

Herralde, F. 2000. Estudio integral de las respuestas ecofisiológicas al estrés hídrico: caracterización de variedades de almendro. Tesis doctoral. Universidad de Barcelona. 
Hoyos, V. 2007. Evaluación y selección de genotipos de Brachiaria spp. por su tolerancia al estrés por sequía y toxicidad por aluminio. Tesis Ingeniero Agrónomo. Universidad de Caldas. Manizales.

Jarma, A., Maza, L., Pineda, A. y Hernandez, J. 2012. Aspectos fisiológicos y bromatológicos de Brachiaria humidicola. Ces. Med. Vet. Zootec

Khan, H., Link, W., Hocking, T., y Stoddard, F. 2007. Evaluation of physiological traits for Características morfo-fisiológicas de frijol común (Phaseolus vulgaris L.) relacionadas con la adaptación a sequía 206 improving drought tolerance in faba bean (Vicia faba L.). Plant Soil. 292:205-217.

\section{Mattos, J., Gomide, J., Martinez, C. y Huaman.} 2005. Crescimento de Espécies do Gênero Brachiaria, sob Déficit Hídrico, em Casa de Vegetação R. Bras. Zootec.34(3):746-754

Mattos, J., Gomide, J. y Martinez, C. 2005. Crescimento de espécies de Brachiaria sob déficit hídrico e alagamento a campo. R. Bras. Zootec.34(3):755-764

Montenegro, G. y Malagón, D. 1990. Propiedades físicas de los suelos Instituto Geográfico Agustín Codazzi. Bogotá. Colombia.

Ontiveros, A., Kohashi, J., Yáñez, P., Acosta, J. y Martínez, A. 2005. Crecimiento de la raíz del frijol con diferentes velocidades de secado del suelo. Terra Latinoamericana 23(3): 311-320.

Passioura, J. 2002. Soil conditions and plant growth. Plant, Cell and Environment. 25(2):311-318.

Reyes, A., Bolaños, E., Hernández, D., Aranda, materia seca y concentración de proteína en 21 genotipos del pasto humidícola Brachiaria humidícola (Rendle) Schweick. Universidad y ciencia, 25(3):213-224.

Rodríguez, A., Cavallini, M. e Cabral, N. 2009. Estresse por Déficit Hídrico em Plantas Forrageiras. Embrapa Caprinos e Ovinos, Brasil. 50 p.

Santos, P., Cruz, P., Araujo, L., Pezzopane, J., Valle, C. and Pezzopane, C. 2013. Response mechanisms of Brachiaria brizantha cultivars to water deficit stress. Revista Brasileira de Zootecnia, 42(11), 767-773.

Scholander, P., Hammel, H. and Bradstreer, Hemmings. 1965. Sap pressure in vascular plants. Science148: 339-346.

Vila, H. 2011. Regulación de la hidratación y la turgencia foliares por mecanismos evitadores del estrés, y resistencia a déficit hídrico en vid Modelo vs. experimentos. Tesis de doctorado. Universidad Nacional de Cuyo, Mendoza.

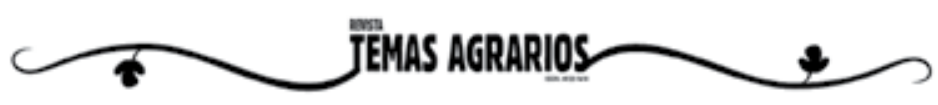

